\title{
NANOSTRUCTURES THERMAL EMISSION OPTIMIZATION USING GENETIC ALGORITHMS AND PARTICLE SWARMS
}

\author{
E. Nefzaoui, J. Drevillon and K. Joulain \\ Institut Pprime, CNRS-Université de Poitiers-ENSMA, Département Fluides, Thermique, Combustion, \\ ESIP-Bâtiment de mécanique, 40, avenue du Recteur Pineau, F 86022 Poitiers, Cedex, France \\ elyes.nefzaoui@univ-poitiers.fr, jeremie.drevillon@univ-poitiers.fr, karl.joulain@univ-poitiers.fr
}

Keywords: particle swarm, genetic algorithm, optimization, coherent thermal emission, nanostructure

\begin{abstract}
Nanotechnologies and nanofabrication techniques provided unmeasureable possibilities to control intrinsic microscopic features of materials and structures in the last years. In particular, materials optical properties and light propagation control have been some of the most challenging problems due to their various application possiblities. The present investigation shows that temporally coherent thermal sources have been successfully designed and optimized with evolutionary optimization methods such as genetic algorithms and particle swarms. This lead to a bilayer structure of germanium and silicon carbide, which is, to our knowledge, the simplest existing structure with such properties.
\end{abstract}

\section{INTRODUCTION}

Thermal sources radiative emission has been for a long time thought to follow the black body laws. This implies emission in quasi all space directions and over a wide wavelengths range. Recently, thanks to advances in materials nanostructuration and related theoretical developments, this paradigm could be surpassed (Greffet and Carminati, 1999; Shchegrov et al., 2000), and sources that exhibit a temporal (temporal coherence is used for monochromatic emission or emission in a very narrow spectral domain) and/or spatial (spatial coherence means emission in specific directions) coherent emission have been designed and fabricated (Sai et al., 2001; Greffet et al., 2002; Richter et al., 1993). This kind of sources are of great interest for new energy conversion devices which aim to improve energy converters such as thermophotovoltaic devices, for radiation detectors and radiative cooling systems. These sources could be realized with various structures exploiting completely different physical phenomena. First, sources of coherent spontaneous emission such as polar materials surmounted by an appropriate surface grating (exploiting surface phonon-polaritons diffraction in the far field) (Greffet et al., 2002) or those based on left-handed materials (artificial materials with a refractive index much lower than 1) (Enoch et al., 2002) emitted essentially in the visible spectrum which is not the wavelength range concerned by thermal emission at usual temperatures (room temperature for example). Later, photonic crystals (PC) (periodic dielectric/metallo-dielectric nanostructures that allow the photons propagation control) made possible the design of infrared (IR) temporally coherent thermal emitters, when introducing a defect in the periodicity of a PC for example (Ben-Abdallah and $\mathrm{Ni}$, 2005). As for spatial coherence, it was obtained with various structures like resonant cavities coupled with metallic layers (Celanovic et al., 2005), surface gratings coupled with waveguides (Joulain and Loizeau, 2007) or a polar material coupled with a semi-infinite PC (Lee et al., 2005; Fu et al., 2005). A general method for spatially and temporally coherent IR thermal sources ab-initio design, based on genetic algorithms, was proposed for the first time by Drevillon (Drevillon and Ben-Abdallah, 2007). This method leads to interesting multilayer structures which could not be found easily by a rational reasoning because of their complexity and random internal structure. In spite of their academic importance, their complexity makes their fabrication at a large scale as difficult as other previously proposed structures such as PC. They though led, after some optimization effort, using par- 
ticle swarm optimization, to very simple structures, bilayer structures in this case, which exhibit very high temporal coherence properties. Theses structures are very interesting since they provide very suitable solutions for industrialization. Besides, their simple internal composition allows an easier analytical approach to understand the underlying physical phenomena of such particular coherence properties.

\section{MULTILAYER STRUCTURES AND GENETIC ALGORITHM OPTIMIZATION}

In this section, a brief presentation of multilayer structures and methods used to calculate their radiative properties is proposed. Their optimization approach with a genetic algorithm is then introduced as well as some of the remarkable obtained results.

\subsection{Multilayer structures}

An example of structures considered in this work is presented in Figure 1. Each structure is composed of $\mathrm{N}$ layers of the same thickness L. Two kinds of structures have been investigated : those with metals and polar materials. Each structure contains at least an absorbent (Silver $(\mathrm{Ag})$, and Silicon carbide $(\mathrm{SiC})$ are used here) and a transparent material (Silicon dioxide $\left(\mathrm{SiO}_{2}\right)$, Silicon $(\mathrm{Si})$ and Germanium $(\mathrm{Ge})$ ) in the considered wavelength range. We are interested in the radiative properties of these structures thus in the spectral and directional emissivity $\varepsilon(\lambda, \theta)$, reflectivity $r(\lambda, \theta)$ and transmittivity $t(\lambda, \theta)$. These quantities are calculated with the basic electromagnetism relations and the whole structure properties obtained with the transfer matrix formalism as detailed by Drevillon (Drevillon, 2007). Simplest obtained structures are compound of 50 layers 50-nanometer thick each. If three different materials are considered in such a structure, more than $7 \times 10^{23}$ different structures are possible. A stochastic method such as genetic algorithms (GA) to explore the search domain is then very useful.

\subsection{Genetic Algorithm}

Since we were looking for a structure with certain emissivity and reflectivity, the following objective

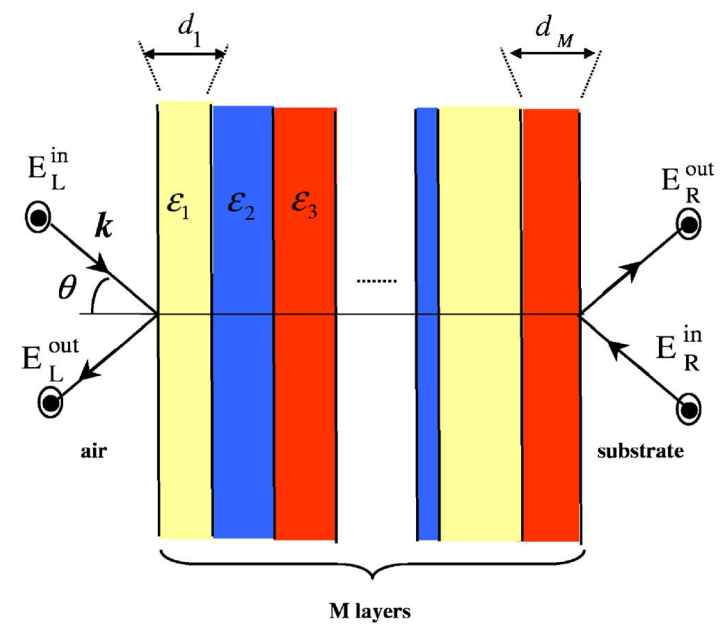

FIG. 1: Schematic of the planar structure made of $\mathrm{N}$ layers of linear dielectrics coated on a semi-infinite substrate. The electric field in out $E L=\left(E L^{\text {in }}, E L^{\text {out }}\right)$ on the left-hand side of the structure is linearly related to the electric field $E R=\left(E R^{\text {out }}, E R^{\text {in }}\right)$ on the right-hand side (Here, only the scattering of TE waves is illustrated. $\mathbf{k}$ is the wave vector of the incident field).

function (fitness) had to be minimized :

$$
\begin{aligned}
J & =\sum_{p} \int_{\theta_{1}}^{\theta_{2}} \int_{\lambda_{\min }}^{\lambda_{\max }}\left[\varepsilon_{\text {target }}(\lambda, \theta)-\varepsilon_{\text {struc }}^{p}(\lambda, \theta)\right]^{2} d \theta d \lambda \\
& +\sum_{p} \int_{\theta_{1}}^{\theta_{2}} \int_{\lambda_{\text {min }}}^{\lambda_{\max }}\left[r_{\text {target }}(\lambda, \theta)-r_{\text {struc }}^{p}(\lambda, \theta)\right]^{2} d \theta d \lambda
\end{aligned}
$$

where the discrete sum operates over both thermal radiation polarization states. $\varepsilon_{\text {target }}$ and $r_{\text {target }}$ are the desired emissivity and reflectivity and $\varepsilon$ and $r$ those of the optimized structure. A simplified version of genetic algorithm was first developed. Considered structures were always made of the same number of layers, and all layers had the same thickness. The only variable parameter to optimize was the different layers order. For instance, if we are in the presence of a two materials structure (material 0 and material 1), it can be represented by a binary sequence of the form $01001101110 \ldots$. A random population is initially generated and the objective function of each individual is then calculated. A pair number of structures among those with the lowest fitness is selected and a crossover is performed between each structures pair. The crossover operation consisted in partially mixing the binary sequences of a pair of structures. For example, the structures $\mathbf{1 1 0 0 1 0 0 1 1}$ and 01011001 are split and recombined to form two children structures $\mathbf{1 1 0 0 1 1 0 0 1}$ and 01010011 . The new generation individuals fitness is calculated and the same ope- 
ration repeated until obtaining a satisfactory fitness. A mutation operator was necessary to avoid convergence to local minima. Some of the obtained results are presented in the following paragraph. A schematic diagram of this algorithm is presented in Figure 2. An initial population of 100 individuals was usually adopted and the stopping criteria was a sufficiently small fitness or a maximal number of iterations (between 100 and 200). Nevertheless, the best structures' fitness varied highly and the algorithm always stopped when reaching the maximal iterations number. A variable mutation probability $p_{m}$ was used and updated at each iteration as detailed in (Drevillon and BenAbdallah, 2007)

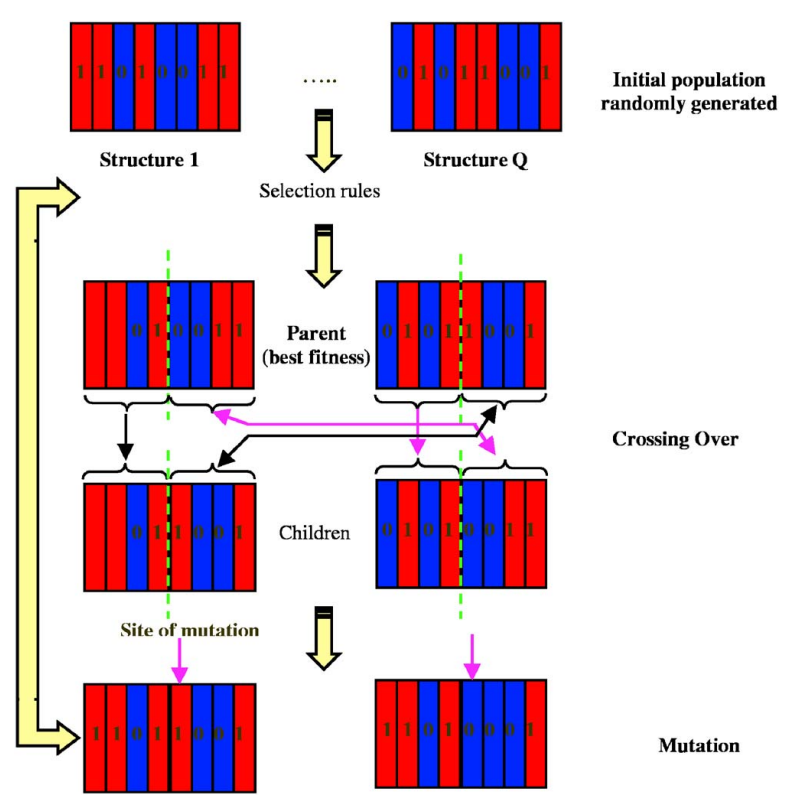

FIG. 2: Principle of genetic algorithm GA used to design a binary nanostructured one-dimensional functional material. Here, the main steps of GA are described : initialization of a random population, selection of parents generation, cross over, and mutation.

\subsection{Results}

First, a temporally coherent thermal source in the wavelength range $[8,14] \mu \mathrm{m}$ using 50 layers of three materials was looked for. In this wavelength domain, Germanium $(\mathrm{Ge})$ and Cadmium Telluride (CdTe) are transparent with approximate dielectric constants $\varepsilon_{G e}=16$ and $\varepsilon_{C d T e}=7.29$ respectively (Palik, 1998). Silicon Carbide $(\mathrm{SiC})$ is the absorbent material. Its dielectric permittivity is well described by the oscil- lating Lorentz's model (Palik, 1998)

$$
\varepsilon_{S i C}=\varepsilon_{\infty}\left(1+\frac{\omega_{L}^{2}-\omega_{T}^{2}}{\omega_{T}^{2}-\omega^{2}-\imath \Gamma \omega}\right)
$$

where $\omega_{L}=18.253 \times 10^{13}{\mathrm{rad} . \mathrm{s}^{-1}}^{-1} \omega_{T}=14.937 \times$ $10^{13} \mathrm{rad} . \mathrm{s}^{-1}, \Gamma=8.966 \times 10^{11}{\mathrm{rad} . \mathrm{s}^{-1}}$ and $\varepsilon_{\infty}=6.7$ are the longitudinal and transverse optical phonon pulsations, the damping factor and the high frequency dielectric constant respectively. A very satisfying structure obtained by this mean is presented in Figure 3. Its directional and spectral emissivity for the TE polarization as well as target emissivity are presented in Figure 4. The obtained source emissivity

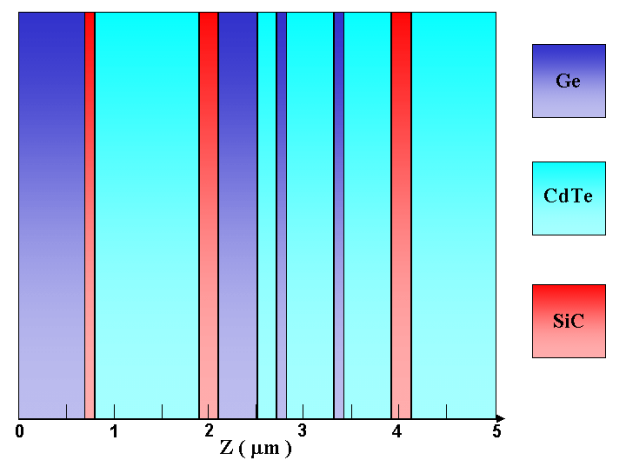

FIG. 3: A schematic of a multilayer structure of $G e, S i C$ and CdTe made of 50 50-nanometer thick layers and obtained by a genetic algorithm in order to have a coherent emission around $\lambda=12.6 \mu \mathrm{m}$.

exhibits a very narrow peak around the wavelength $\lambda_{m}=12.6 \mu \mathrm{m}$ that culminates at $\varepsilon_{\text {max }}=0.9$ for both polarizations. However, a degradation of the emission coherence occurs at large angles for TM polarization. The obtained source radiative properties are very interesting, since they present the highest coherence degrees ever observed with thermal sources at this wavelength range. However, its structure is still quite complex from an industrial point of view and still needs further improvements. In the following section, another optimization method is adopted, and we show that it allowed the design of structures as simple as a bilayer and with coherence degrees even higher than those of the structure aforementioned.

\section{BILAYER STRUCTURES AND PARTICLE SWARMS OPTIMIZATION (PSO)}

Starting from the structures obtained with GA and with a manual trial and error approach, it has been 

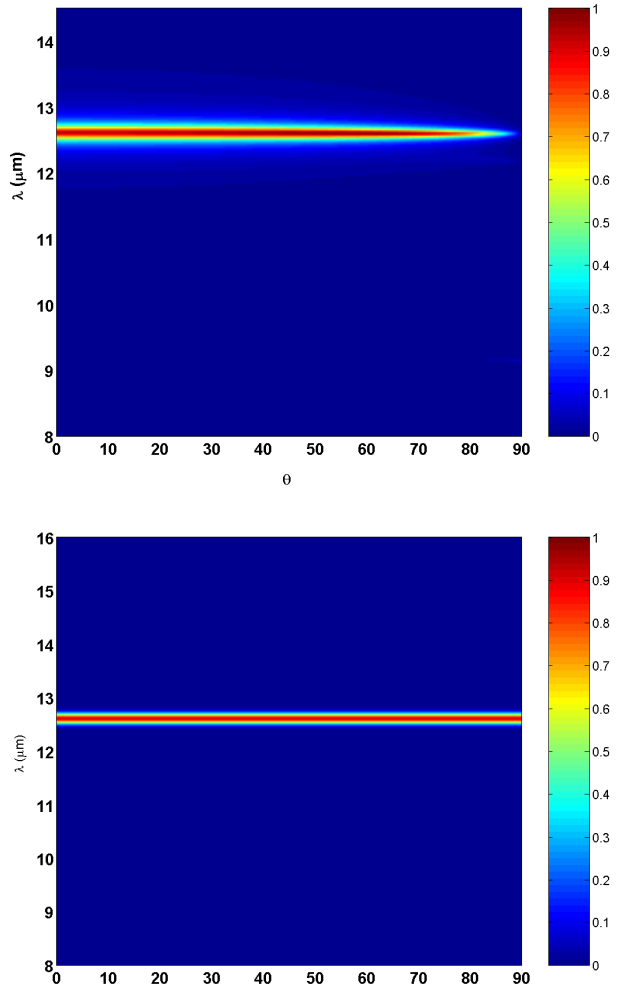

FIG. 4: Spectral and directional emissivity (TE polarization) of the multilayer structure presented in Figure 3 and target emissivity adopted in the optimization process by the GA.

shown that simpler structures with a high coherence degree were realizable. A PSO algorithm was used to optimize them taking into account more parameters like dielectric permittivities and layers thicknesses which allowed to understand their influence on such radiative properties and the underlying physical phenomena. Finally, the design of other IR coherent thermal sources with different materials and over other wavelength domains was made possible. These different steps and obtained results are presented in the following paragraphs.

\subsection{Trial and error approach}

Even if the structure presented in Figure 3 is made of 50 elementary layers, it is obvious that this number can be reduced thanks to the existence of adjacent layers of the same materials. According to Figure 3 it can be reduced to at least 13, without changing the global structure. After more than 35 trials and errors, where layers thicknesses, the number of layers, and their organization were modified manually, a sim- pler coherent source could be obtained by eliminating $C d T e$ and using only $G e$ and $\mathrm{SiC}$. This structure is presented in Figure 5 and its directional and spectral emissivity and reflectivity are plotted in Figure 6. It is

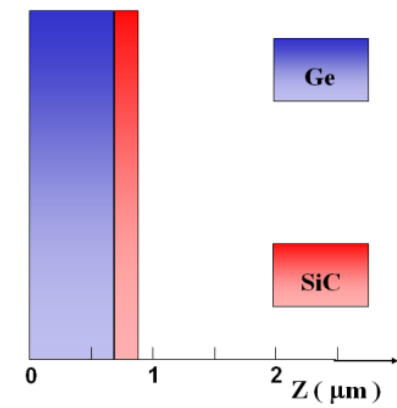

FIG. 5: Bilayer structure made of a 700nm thick $G e$ layer and a $200 \mathrm{~nm}$ thick $\mathrm{SiC}$ layer. This structure was obtained when trying to simplify manually the 50 layers structure obtained by GA while keeping high coherence and emission properties.
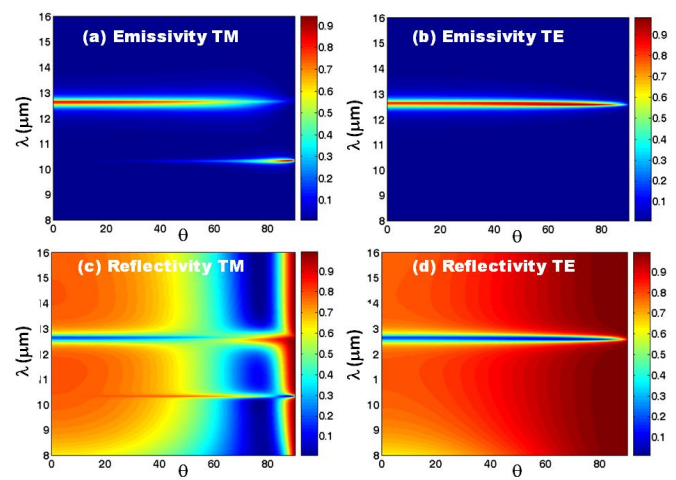

FIG. 6: Spectral and directional emissivity for TM (a), TE (b) polarizations, and reflectivity for TM (c), TE (d) polarizations of the quasimonochromatic thermal source presented in Figure 5.

clear that coherence properties, compared to those of the multilayer structure of Figure 3 are improved. The trial and error process does not allow to find the optimal structure since many local minima exist around the global minimum. Founding the global minimum this way would at least necessitate a big number of off-putting essays. We also wanted to know whether it was possible to obtain coherent sources with other materials. A PSO algorithm was then used.

\subsection{PSO and Bilayer optimization}

To determine the optimal bilayer structure with $\mathrm{SiC}$ and $G e$ or any other transparent material and in or- 
der to know whether other coherent emitting structures with different materials were possible, we had to explore a search space with more dimensions. We then adopted PSO and looked for the optimal layers thicknesses and materials dielectric permittivities. We although kept the same form of the absorbent material dielectric permittivity (Lorentz oscillator). Particles were initialized with random positions $\left(x_{i}\right)$ and nil velocities $\left(v_{i}\right)$. Then, they were updated at each iteration as following :

$$
\begin{gathered}
v_{i}=\omega v_{i}+c_{1} r_{1}\left(x_{b n, i}-x_{i}\right)+c_{2} r_{2}\left(x_{b m, i}-x_{i}\right)(3) \\
x_{i}=x_{i}+v_{i}
\end{gathered}
$$

where $\omega=0.729$ is inertia weight factor, $r_{1}$ and $r_{2}$ are two random factors, $c_{1}=1.494$ and $c_{2}=1.494$ are the "social" and "cognitive" weight factors, $x_{b n, i}$ and $x_{b m, i}$ the best positions in the particle $i$ neighborhood and memory respectively. Populations were always composed of 20 particles. 1000 iterations were sufficient to obtain the best structures. Finally, since physical parameters are looked for, all values are not allowed for the optimized parameters. When a particle run out of the search domain, it was put back on its boundary with a nil velocity. This procedure showed that for $\mathrm{SiC}$ and $\mathrm{Ge}$ structures, the optimum is obtained for slightly different thicknesses $\left(d_{S i c}=65 \mathrm{~nm}\right.$ and $d_{G e}=735 \mathrm{~nm}$ ) and that it is possible to design coherent thermal sources with other materials. The resulting $\mathrm{Ge}-\mathrm{SiC}$ structure is presented in Figure 7 and its radiative properties in Figure 8. Thanks to the sim-

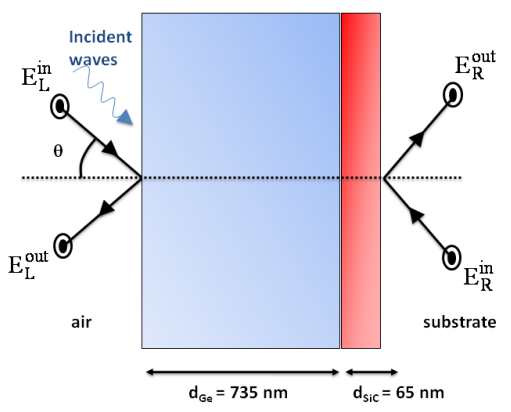

FIG. 7: Optimal quasimonochromatic structure made of a $735 \mathrm{~nm}$ thick $\mathrm{Ge}$ layer and a $65 \mathrm{~nm}$ thick $\mathrm{SiC}$ layer. This structure was obtained when trying to improve the previously and manually obtained bilayer structure (Figure 5) using PSO. This structure presents the highest emission intensity while conserving the best coherence level.

plicity of bilayer structures, an analytical approach is
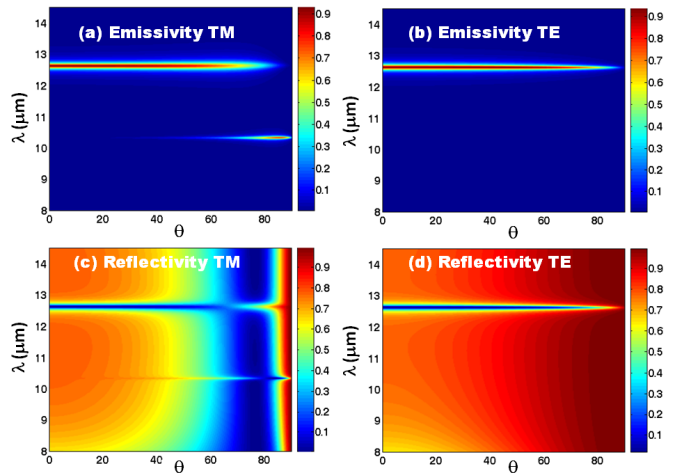

FIG. 8: Spectral and directional emissivity for TM (a), TE (b) polarization, and reflectivity for TM (c), TE (d) polarization of the optimal quasimonochromatic thermal source obtained by PSO and presented in Figure 7.

possible. The reflectivity of such a structure is given by :

$$
r e^{l \delta}=\frac{r_{12}+r_{23} e^{-l \Delta_{2}}+r_{34} e^{-l\left(\Delta_{2}+\Delta_{3}\right)}+r_{12} r_{23} r_{34} e^{-i \Delta_{3}}}{1+r_{12} r_{23} e^{-l \Delta_{2}}+r_{12} r_{34} e^{-l\left(\Delta_{2}+\Delta_{3}\right)}+r_{23} r_{34} e^{-i \Delta_{3}}}
$$

where

$$
\begin{aligned}
& r_{12}=\frac{n_{1}-n_{2}}{n_{1}+n_{2}} \\
& r_{23}=\frac{n_{2}-\tilde{n_{3}}}{n_{2}+\tilde{n_{3}}} \\
& r_{34}=\frac{\tilde{n_{3}}-n_{4}}{\tilde{n_{3}}+n_{4}}
\end{aligned}
$$

are Fresnel reflexion coefficients between media 1-2, 2-3 and 3-4 respectively and

$$
\begin{aligned}
\Delta_{2} & =\frac{4 \pi d_{2} n_{2}}{\lambda} \\
\Delta_{3} & =\frac{4 \pi d_{3} \tilde{n_{3}}}{\lambda}
\end{aligned}
$$

are the electromagnetic wave phase shifts due to the propagation through media 2 and 3 respectively. $d_{2}$ and $d_{3}$ are the thicknesses of the layers 2 and $3, n_{1}$, $n_{2}, \tilde{n_{3}}$ and $n_{4}$ are the refractive indices of the media 1 (air), 2(Ge), 3(SiC) and 4(air). The tilde on $\tilde{n_{3}}$ means that this quantity is complex since $\mathrm{SiC}$ is an absorbent medium in the considered wavelength range. We realized that for thicknesses given by PSO, the structure reflectivity goes to zero at the emission peak wavelength and is very high for other wavelengths. This means that $G e$ layer plays the role of 


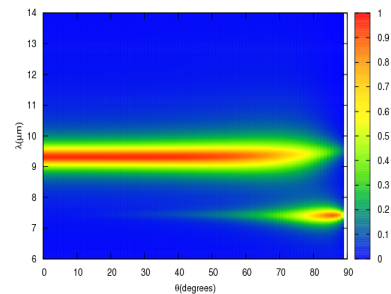

(a) Emissivity TE

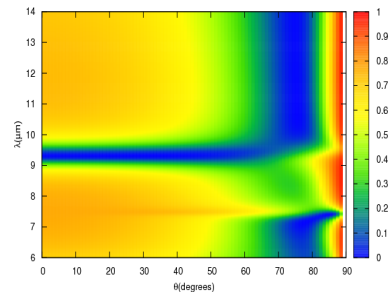

(c) Reflectivity TE

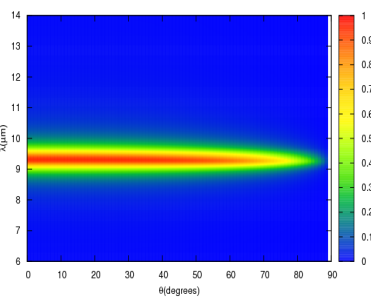

(b) Emissivity TM

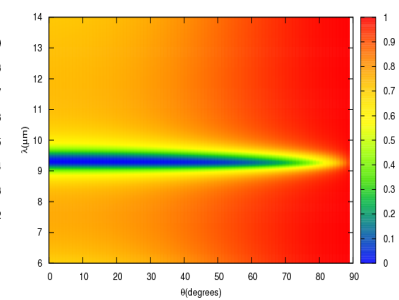

(d) Reflectivity TM

FIG. 9: Spectral and directional emissivity and reflectivity for TM and TE polarizations of a bilayer structure made of a $450 \mathrm{~nm}$ thick $G e$ layer and a $310 \mathrm{~nm}$ thick $B N$ layer.

an antireflector for a certain wavelength. This is possible because $\mathrm{Ge}$ layer thickness, $\mathrm{Ge}$ and $\mathrm{SiC}$ dielectric permittivities satisfy particular conditions(Zhang, 2007). Besides, SiC dielectric permittivity resonance occurs at this same wavelength. SiC layer is then excited at its resonance which leads to the high emissivity. For other wavelengths incident radiation is reflected and can not reach $\mathrm{SiC}$ layer. In addition, the highest coherence degree and emission intensity are obtained only for $d_{S i C}=65 \mathrm{~nm}$. In fact, $S i C$ layer also plays a waveguide role. For higher thicknesses, more modes around the resonance mode can propagate which leads to a coherence loss. For lower thicknesses, the structure is not opaque anymore since the absorbent layer is then thinner than the penetration depth. An increase of transmittivity leads to a loss in the emissivity intensity. Taking into account these different observations which have been made possible by the simplicity and accuracy of PSO results, other coherent thermal sources could be designed. For instance, a bilayer structure constituted of a $450 \mathrm{~nm}$ thick $G e$ layer and a $310 \mathrm{~nm}$ thick boron nitride $(B N)$ layer presents original coherence properties around $\lambda=9.3 \mu \mathrm{m}$. $B N$ dielectric permittivity is modeled by a Lorentz oscillator (Palik, 1998). This structure emissivity for TM polarization is given in Figure 9.

Due to the form of the dielectric permittivity of $B N$ whose peak is larger than $\mathrm{SiC}^{\prime}$ 's one, this source

is less coherent than those based on SiC. However, it shows that it is possible to obtain other coherent thermal sources based on the same principle, with other materials and on other wavelength ranges. It also gives interesting ideas to greatly improve theses sources coherence by controlling and tuning the polar materials (such as $\mathrm{SiC}$ and $\mathrm{BN}$ ) dielectric permittivities (making the peaks narrower for example).

\section{CONCLUSIONS}

It was shown in this paper that evolutionary optimization methods (genetic algorithms and particle swarms) can be very helpful for the inverse design of complex nanostructures with peculiar properties. It was possible to design multilayer nanostructures with coherent thermal emission in the mid infrared region in spite of a relative ignorance of the precise physical phenomena underlying such properties. The same optimization methods helped in simplifying these sources to finally obtain $G e-S i C$ bilayer structures, which are, to our knowledge, the simplest existing structures with such features. The simplicity of the resulting objects, made possible an analytical study and the understanding of their internal functioning. This allows a more rational design of of new coherent thermal sources emitting in other wavelength ranges. Optimization algorithms are being improved to implement multi-objective optimization and make more systematic the design and simplification of thermal sources in mid and near infrared regions, with a big variety of materials.

\section{ACKNOWLEDGMENTS}

Elyes Nefzaoui thanks Jacques Nérault, Manuel Girault and Younes Ezzahri for helpful discussions.

\section{REFERENCES}

Ben-Abdallah, P. and Ni, B. (2005). Single-defect bragg stacks for high-power narrow-band thermal emission. J. Appl. Phys., $97: 104910$.

Celanovic, I., Perreault, D., and Kassakian, J. (2005). Resonant-cavity enhanced thermal emission. Phys. Rev. $B, 72$ :075127.

Drevillon, J. (2007). Design ab-initio de matériaux micro et nanostructurés pour l'émission thermique cohérente en champ proche et en champ lointain. $\mathrm{PhD}$ thesis, Université de Nantes. 
Drevillon, J. and Ben-Abdallah, P. (2007). Ab initio design of coherent thermal sources. J. Appl. Phys., $102: 114305$.

Enoch, S., Tayeb, G., Sabouroux, P., Guérin, N., and Vincent, P. (2002). A métamaterial for directive emission. Phys. Rev. Lett., $89: 213902$.

Fu, C. J., Zhang, Z. M., and Tanner, D. B. (2005). Planar heterogenous structures for coherent emission of radiation. Opt. Lett., $30: 1873$.

Greffet, J. J. and Carminati, R. (1999). Near-field effects in spatial coherence of thermal sources. Phys. Rev. Lett., $82: 1660-1663$.

Greffet, J. J., Carminati, R., Joulain, K., Mulet, J. P., Mainguy, S., and Chen, Y. (2002). Coherent emission of light by thermal sources. Nature, $416: 61-64$.

Joulain, K. and Loizeau, A. (2007). Coherent thermal emission by microstructured waveguides. JQSRT, $104: 208$.

Lee, B. J., Fu, C. J., and Zhang, Z. M. (2005). Coherent thermal emission from one-dimensional photonic crystals. Appl. Phys. Lett., 87 :071904.

Palik, E. D. (1998). Handbook of optical constants of solids. Academic Press, London.

Richter, K., Chen, G., and Tien, C. L. (1993). Partial coherence theory of multilayer thin-film optical properties. Opt. Eng., 32 :1897-1903.

Sai, H., Yugami, H., Akiyama, Y., Kanamori, Y., and Hane, K. (2001). Spectral control of thermal emission by periodic microstructured surfaces in the near-infrared region. J. Opt. Soc. Am. A, 18 :1471-1476.

Shchegrov, A. V., Joulain, K., Carminati, R., and Greffet, J. J. (2000). Near field spectral effect due to electromagnetic surface excitations. Phys. Rev. Lett., 85 :1548-1551.

Zhang, Z. M. (2007). Nano/microscale Heat Transfer. McGraw-Hill, New York. 